\title{
Invasive prenatal diagnostic procedures: a developing countries' perspective
}

\author{
Namrata Kashyap*, Mandakini Pradhan, Sangeeta Yadav, Neeta Singh
}

\begin{abstract}
Department of Maternal and Reproductive Health, Sanjay Gandhi Post Graduate Institute of Medical Sciences,
\end{abstract} Lucknow, India

Received: 25 November 2015

Accepted: 12 December 2015

\section{*Correspondence:}

Dr. Namrata Kashyap,

E-mail: dr.nmrata@gmail.com

Copyright: (c) the author(s), publisher and licensee Medip Academy. This is an open-access article distributed under the terms of the Creative Commons Attribution Non-Commercial License, which permits unrestricted non-commercial use, distribution, and reproduction in any medium, provided the original work is properly cited.

\begin{abstract}
Background: Antenatal procedures are effective prenatal diagnostic tool for detection of fetal disorders. They have been practiced since time long, still in developing countries like India they are yet to find a place. Here, we report our experience with antenatal procedures from a single medical centre, focusing on the indications and outcome of invasive prenatal procedures.

Methods: This is retrospective observational study was conducted on pregnant women presenting at Sanjay Gandhi Post Graduate Institute of Medical Sciences; Lucknow, India between July 2009 and September 2013 was conducted. The data were analyzed to find out the indications, gestational age, complications and outcome of diagnostic prenatal testing.

Results: Antenatal diagnostic procedures include amniocentesis, chorionic villous sampling, cordocentesis, vesicocentesis and paracentesis. There were 473 total number of procedures done during this period, of which 53 (11.2\%) were CVS, 315 (66.5\%) were amniocentesis, 72 (15.2\%) were cordocentesis, 21(4.4\%) were vesicocentesis and $7(1.4 \%)$ were paracentesis. Out of total procedures $47(9.9 \%)$ procedures results were abnormal while 426 had normal results. In abnormal result group, 24 patients (51\%) were of gestational age of less than or equal to 20 weeks. All those with lethal / major malformation underwent termination of pregnancy where gestational age of less than 20 weeks.

Conclusions: With appropriate prenatal invasive test were able to prevent birth of affected fetus which is of huge importance considering the patients who give birth to abnormal babies only to see them suffering and frequently dying also. Prenatal invasive test were able to prevent this psychological, mental as well as physical trauma in these patients.
\end{abstract}

Keywords: Prenatal, Amniocentesis, CVS

\section{INTRODUCTION}

Prenatal diagnosis refers to part of fetal medicine meant to determine the health condition of an unborn foetus. In absence of prior knowledge gained by prenatal diagnosis, there could be an untoward outcome for the foetus or the mother or both. Congenital anomalies account for 20 $25 \%$ of perinatal deaths and chromosomal abnormalities account for $5 \%$ of still birth. Antenatal procedures are effective prenatal diagnostic tool for detection of foetal disorders. The American college of Obstetrics and Gynaecology recommends that all women irrespective of age should be subjected to screening for aneuploidy. ${ }^{1}$ Present time obstetric care therefore includes noninvasive screening approaches as well as invasive procedures for the definitive prenatal diagnosis of foetal disorders, correlations between indications for prenatal cytogenetic diagnosis and results of the chromosomal analysis made upon foetal cells. 
The two most common procedures involved are amniocenteses and chorionic villous sampling. Amniocentesis is performed to obtain amniotic fluid for karyotyping from 15 weeks on wards. Amniocentesis performed before 15 completed weeks of gestation is referred to as "early amniocenteses". Chorionic villous sampling (CVS) is usually performed between 11 and 13 weeks of gestation and involves aspiration or biopsy of placental villi. CVS can be performed using either a transabdominal or a transcervical approach. The complication varies from procedure to procedure.

Prenatal invasive procedures are not a new invention. They have been practised since time long, still in developing countries like India they are yet to find a place. There are countable centres in the country where these procedures are carried out. To justify their importance and with their growing use, need arises to audit these procedures, to observe the benefit versus the associated risks. Here, we report our experience with antenatal procedures from a single medical centre, focusing on the benefits achieved with these invasive prenatal procedures. The study was planned to analyse the various indications, complications and benefits of prenatal diagnostic procedures in a tertiary care centre.

\section{METHODS}

This was a single centre, retrospective observational study of 4 years. Antenatal procedures (amniocentesis, chorionic villous biopsy, cordocentesis, paracentesis and vesicocentesis) on pregnant women presenting at Department of Maternal and Reproductive Health, Sanjay Gandhi Post Graduate Institute of Medical Sciences, Lucknow, India from June 2009 and June 2013 were analysed. Indications, timing, complications and outcome of these procedures were evaluated focussing on the need, availability and benefits of these procedures. Descriptive proportions and frequencies have been used to depict the data.

Out of a total of 4080 pregnant women attending the outpatient department, there were 473 total number of procedures done, of which $53(11.2 \%)$ were CVS, 315 $(66.5 \%)$ were amniocentesis, $72 \quad(15.2 \%)$ were cordocentesis, $21(4.4 \%)$ were vesicocentesis and 7 (1.4\%) were paracentesis (Table 1).

Table 1: Various types of invasive procedures.

\begin{tabular}{|lll|}
\hline Type of procedure & Number & Percentage \\
\hline Amniocentesis & 315 & $66.5 \%$ \\
\hline Cordocentesis & 72 & $15.2 \%$ \\
\hline Chorionic villous sampling & 53 & $11.2 \%$ \\
\hline Vesicocentesis & 21 & $4.4 \%$ \\
\hline Paracentesis & 7 & $1.4 \%$ \\
\hline Diagnostic amnioinfusion & 3 & $0.6 \%$ \\
\hline $\begin{array}{l}\text { Aspiration from cystic } \\
\text { hygroma }\end{array}$ & 2 & $0.4 \%$ \\
\hline Total procedures & 473 & \\
\hline
\end{tabular}

The indications for amniocentesis were raised risk for trisomy 21 in serum screening (112), abnormal genetic sonogram (180), parents with abnormal karyotype (5), previous baby with chromosomal abnormality or family history of chromosomal or single gene disorder (8), and suspected fetal infections (10).

Out of 53 patients undergoing chorionic villous sampling, 18 had previous history of baby affected with chromosomal abnormality, genetic abnormality (cystic fibrosis, thalassemia, and hemophilia), and single gene disorders like Duchene muscle dystrophy, sickle cell disease, congenital adrenal hyperplasia, spinal muscle atrophy and achondroplasia. Additional 27 patients had both parent's trait for thalassemia or sickle cell disease. Rest 8 patients had sonographic abnormality like suspected triploidy (placentomegaly, IUGR and bradycadia), cystic hygroma, hydrops and raised nuchal thickness more than $3.5 \mathrm{~mm}$.

The indications for cordocentesis were suspected fetal anaemia diagnosed through raised Middle cerebral artery peak systolic velocity (8), previous baby with chromosomal abnormality or family history of chromosomal or single gene disorder (10), abnormal genetic sonogram (52) (Table 2).

Table 2: Various indications for the procedure.

\begin{tabular}{|llll|}
\hline Indications & Amniocentesis & $\begin{array}{l}\text { Cordocentesi } \\
\text { CVS }\end{array}$ \\
\hline Raised risk in serum screening more than 1 in 100 after modification & 112 & - & - \\
\hline USG abnormality & 180 & 60 & 8 \\
\hline Single gene disorder / genetic / chromosomal abnormality & 8 & 10 & 45 \\
\hline Suspected fetal infection & 10 & 2 & - \\
\hline Parents with abnormal karyotype & 5 & - & - \\
\hline Total & 315 & 72 & 53 \\
\hline
\end{tabular}


The indications for vesicocentesis included fetus with sonographic findings suggestive of urinary tract obstruction like posterior urethral valve in eight foetuses and urethral atresia in three foetuses and one fetus each of megacystis and urorectal septal malformation.

The indications for paracentesis were isolated urinary ascites in four and isolated fetal ascites in three foetuses.
Out of 473 patients, $284(60 \%)$ patients presented at gestational age $\leq 20$ weeks and 151 (40\%) patients presented after 20 weeks.

All CVS were done between 8-28 weeks, amniocentesis between 14- 33 weeks, cordocentesis between $22-33$ weeks and vesicocentesis between 18 - 28 weeks of gestational age (Table 3).

Table 3: Gestational age at which the procedure was carried out.

\begin{tabular}{|llll|}
\hline Gestational age & Amniocentesis & Cordocentesis & CVS \\
\hline$\leq 20$ weeks & 229 & 8 & 47 \\
\hline Normal & 216 & 5 & 39 \\
\hline Abnormal & 13 & 3 & 8 \\
\hline$\geq 20$ weeks & 86 & 59 & 6 \\
\hline Normal & 72 & 54 & 2 \\
\hline Abnormal & 14 & 5 & 4 \\
\hline
\end{tabular}

\section{RESULTS}

Out of total procedures, $47(9.9 \%)$ procedure results were abnormal while 426 had normal results (Table 5). In abnormal result group, 24 patients $(51 \%)$ were of gestational age of $\leq 20$ weeks. All those with lethal / major malformation underwent termination of pregnancy where gestational age of less than 20 weeks (Table 4).

Table 4 : Various complications associated with the procedures.

\begin{tabular}{|lllll|}
\hline Complications & Amniocentesis 315 & Cordocentesis 72 & CVS 53 & Others 23 \\
\hline Spontaneous abortion & 0 & 0 & 0 & 0 \\
\hline Preterm labour & 1 & 0 & 0 & 0 \\
\hline Preterm premature rupture of membranes & 1 & 0 & 0 & 0 \\
\hline Chorioamnionitis & 0 & 0 & 0 & 0 \\
\hline Intrauterine fetal death & 0 & 1 & 0 & 0 \\
\hline Procedure failure & 0 & 0 & 0 & 0 \\
\hline Culture failure ( 12, 2.5\%) & 8 & 2 & 1 & 1 \\
\hline
\end{tabular}

Out of total 21 cases of vesicocentesis, total 19 procedures were done for posterior urethral valves for with diagnostic and therapeutic intent. One patient had isolated megacystis. Megacystic microcolon syndrome was ruled out and serial reduction was done to relieve the backpressure and thus reflux nephropathy was avoided. Patient had full term ceasarean section. Baby and postnatal period was uneventful. Other patient had uroseptal malformation which was confirmed only when distended fluid in common chamber was reduced to optimize visualization of other abnormalities (Table 5).

There were seven cases with fetal ascites, four had urinary ascites which was confirmed with biochemical examination and three had isolated fetal ascites. For these three foetuses, diagnosis was evident on gross examination. In two cases fluid was white coloured suggestive of chylous ascites and in one case fluid was green coloured suggestive of meconium peritonitis (Table $6)$.

Instillation of saline as a part of diagnostic amnioinfusion was done in three patients and it helps to reach the diagnosis of limb body wall complex, bilateral multicystic dysplastic kidney and urorectal septal malformation.

Various post procedure complications were also noted which included spontaneous abortion, preterm labour, infections, fetal death procedure and culture failure (Table 7). 
Table 5: Diagnostic information achieved with invasive procedures.

\begin{tabular}{|c|c|c|c|}
\hline \multirow{10}{*}{ Abnormal karyotype } & \multirow[t]{10}{*}{35} & Trisomy 21 & 12 \\
\hline & & Trisomy 18 & 4 \\
\hline & & Trisomy 13 & 2 \\
\hline & & Turner's & 4 \\
\hline & & Triploidy & 1 \\
\hline & & Addition $6 \mathrm{q}$ & 1 \\
\hline & & Prominent satellites 15,13 & 2 \\
\hline & & Hetrochromatin & 2 \\
\hline & & XXY & 1 \\
\hline & & Inversions, translocation & 3 \\
\hline Congenital rubella & 1 & & \\
\hline Fetal hypothyroidism & 1 & & \\
\hline Fetal anemia non isoimmunized & 2 & & \\
\hline \multirow[t]{5}{*}{ Single gene disorders } & \multirow[t]{5}{*}{10} & Duchene muscle dystrophy & 1 \\
\hline & & Congenital adrenal hyperplasia & 1 \\
\hline & & Hemophillia & 1 \\
\hline & & $\begin{array}{l}\text { Thalassemia major } \\
\text { ( compound hetrozygous) }\end{array}$ & 5 \\
\hline & & Sickle cell disease & 2 \\
\hline
\end{tabular}

Table 6: Information and benefits with fetal vesicocentesis.

\begin{tabular}{|lll|}
\hline Vesicocentesis & 21 including 8 repeat procedures \\
$\begin{array}{l}\text { Lower urinary tract obstruction } \\
\text { 1. Posterior urethral valve }\end{array}$ & 11 & 5 fetuses survived who underwent \\
postnatal surgery for posterior \\
2. Posterior urethral valve with grade 3-4 hydronephrosis & 3 & $\begin{array}{l}\text { urethral valve are on follow up . } \\
2 \text { amongst them are on treatment of } \\
\text { vescicoureteric reflux. }\end{array}$ \\
\hline Megacystis & 8 & Good outcome \\
\hline Urorectal septal malformation & 1 & terminated \\
\hline
\end{tabular}

Table 7: Outcome with fetal paracentesis.

\begin{tabular}{|lll|}
\hline Paracentesis & 7 & Findings \\
\hline Urinary ascites & 4 & suggestive of \\
1. Posterior urethral valve with grade III - IV hydronephrosis & 3 & urinary ascites \\
2. Meatal stenosis & 1 & 3 \\
\hline Isolated fetal ascites & 2 (triglycerides lymphocytes) \\
1. Chylous ascites & 1 (meconium green coloured) \\
2. Meconium peritonitis & a & \\
\hline
\end{tabular}

\section{DISCUSSION}

Prenatal invasive diagnosis refers to invasive testing for diseases or conditions in a fetus or embryo in utero, before birth with intent to detect birth defects, chromosomal abnormalities and genetic diseases which lead to death or severe handicap or if fetus appears salvageable, necessary prenatal measures could arranged prior to delivery. Prenatal invasive procedures include amniocentesis, chorionic villous biopsy, cordocentesis (percutaneous umbilical cord blood sampling) and any other procedure to obtain fetal sample like fluid aspirated from distended bladder (vesicocentesis), fetal peritoneal cavity (paracentesis) or hygroma fluid aspiration.

Majority of the times, these tests are administered to determine if the fetus needs to be aborted but if it is beyond the limit and capacity of termination, it useful to diagnose high-risk pregnancies and foetuses early so that delivery can be scheduled in a tertiary care hospital where 
the baby can receive appropriate care and health care staff can be better prepared for postnatal management of the baby with a known health problem.

Amniocentesis can be done from about 14 weeks gestation, and usually up to about 20 weeks, and chorionic villous sampling can be done earlier (between 9.5 and 12.5 weeks gestation) but may be slightly more risky to the fetus. Evan et al in their study compared transabdominal chorionic villous sampling with second trimester amniocentesis and found no significant difference in the total pregnancy loss between the two procedures. $^{2}$ The best estimate of miscarriage risk associated with amniocentesis comes from a randomized trial from Denmark reported in 1986. ${ }^{3}$ This study randomized 4606 pregnant women at low risk of miscarriage aged 24-35 years to have or not to have an amniocentesis carried out using a 20-gauge needle under continuous real-time ultrasound guidance. Most procedures were performed between 16-18 weeks of gestation. The amniocentesis group had a loss rate which exceeded the control group by $1 \%$. More than $50 \%$ of the amniocenteses were performed by one operator and the remainder by four other operators who were less experienced. The placenta was avoided whenever possible but a transplacental approach was recorded in $15 \%$ of cases. Bloodstained amniotic fluid was obtained in $0.5 \%$ of cases overall. The compilation of published data suggests that procedure risks of amniocentesis to be about $1 / 200$ and slightly lower in very skilled hands. The risks of CVS in very experienced hands may also be about 1/200. Prospective comparative studies have demonstrated that with equally experienced operators, CVS and second trimester amniocentesis have similar procedure induced miscarriage rates. When CVS procedures are performed after 10 weeks gestation, no increased risk of fetal anomalies has been demonstrated. ${ }^{4}$

So we realize that contrary to the common belief of CVS being a risky procedure than amniocentesis, most studies including our study shows that comparing CVS to amniocentesis in skilled hands have found equivalency of risks. We have not practiced transcervical CVS in a comparable number of patients but several randomized trials show almost identical miscarriage rates after transcervical CVS compared with the transabdominal approach. ${ }^{5,6}$ It is important to consider is that there is no increased risks of limb reduction defects following CVS at 10 weeks or greater, but there is an increase in Talipes from early amniocentesis. Hence in the first trimester Chorionic villous Sampling appears to be a safer procedure. Also a point to highlight is that meta-analysis comparing transcervical CVS with second trimester amniocentesis showed that amniocentesis is significantly safer. ${ }^{7}$

When we talk about risk associated with the procedure we must also consider the fact the risk varies not only between procedures but also the indications for which the procedure is being done. Rashmi Nanal et al in their study reinforced that pregnancy loss rates after prenatal invasive procedures such as amniocentesis, chorionic villous sampling and fetal blood sampling (FBS) vary with an underlying indication for the procedure and due to different background risk of miscarriage. ${ }^{8}$ The procedure-related pregnancy loss rate was obtained by subtracting the losses in pregnancies with known lethal conditions and those occurring more than two weeks after the procedure from total pregnancy losses. The total pregnancy loss rate was $4.1 \%$ and the procedure-related loss rate was $0.23 \%$ (95\% CI, 0.0061-1.28). The procedure-related loss rates for Amniocentesis was $0.7 \%$ (95\% CI, 0.2-1.8) and FBS was 1.19 (95\% CI, 0.144.23).

In our study, out of a total 473 procedure over a period of 4 years, the overall complication rate came out as 0.0029 $\%$, a risk less than 1 per 1000 . No well controlled studies as yet support claims of amniocentesis related risk at $1 / 1000$ or lower. Also whatever pregnancy risk or failure of culture we have noticed was very highly dependent on pre procedure scenario rather than operator experience. There was one case of preterm premature rupture of membranes with preterm delivery where amniocentesis was done at 32 weeks for a fetus which showed congenital diaphragmatic hernia along with transposition of great vessels and severe polyhydramnious.

There were 12 patients who had culture failure, eight with amniocentesis (one patient each of molar pregnancy, posterior urethral valve, holoprosencephaly and 5 patients with sonographic soft markers of aneuploidy), two in cordocentesis and one each with CVS and cystic hygroma aspiration. In CVS group there was one patient with raised risk with trisomy 21 along with previous thalassemia) while in cordocentesis there were two failures with patient showing USG abnormality (both had Oligohydraminous and posterior placenta were fetal sample was achieved less satisfactorily). There were two cases of intrauterine death following cordocentesis for which the cause could not be ascertained; however it could be attributed to fetal vasospasm.

Being a study from Indian population, prenatal invasive procedures helped in termination of 24 fetuses amongst 47 abnormal $(51 \%)$ within medico legal limit. ${ }^{9}$ This is an important aspect as it avoids the untoward forced illegal acts and reduced maternal morbidity and mortality when the termination is carried out at substandard places.

The burden of managing and care of child suffering from chromosomal abnormality can be estimated by available evidences in literature. ${ }^{10-12}$ These results suggest that genetic disease, although less frequent than disease caused by environmental conditions, lays a considerable financial burden on the hospital and on the affected families. The need to create genetic units in developing countries is emphasized. We find it worth to prevent birth of 27 fetuses with abnormal karyotype. Diseases like thalassemia major and sickle cell disease pose a 
significant burden of blood transfusion and medication. Throughout the lifetime the child is either dependent on blood transfusion or needs transplantation. Very frequently these children succumb to various illnesses as a spectrum of the disease. Henry et al showed the role and ease of amniocentesis in the intrauterine detection of genetic disorders. ${ }^{13}$ One hundred and sixty two transabdominal amniocenteses were performed between 13-18 weeks of fetal gestation as part of the management of 155 high-risk pregnant women. Successful cultivation of amniotic-fluid cells led to the intrauterine detection of Down's syndrome (10 cases), Pompe's disease (1 case), lysosomal acid phosphatase deficiency (1 case) and metachromatic leukodystrophy (1 case). They also believed that the risk of this procedure is low since neither fetal nor maternal complications were demonstrated in this series of patients. Cultivation of amniotic-fluid cells obtained by transabdominal amniocentesis early in the second trimester of pregnancy provides a method that enables parents at high risk for having offspring with certain serious genetic disorders to have children free of risk of such abnormalities.

Daniela Neagos et al confirmed the importance of screening and the cytogenetic diagnosis in the identification of the numerical chromosomal abnormalities. ${ }^{14}$ Amniotic fluid samples from 1159 pregnant women were studied with rapid fish method and the cytogenetic analysis (karyotype) to diagnose numerical chromosomal abnormalities in cases of abnormal results of double or triple test, advanced maternal age, fetal abnormality detected through ultrasound examination, and positive family history for chromosomal anomalies. In their study they identified 30 cases with abnormal numeric chromosomes (18 cases of trisomy 21,4 cases of trisomy 18,3 cases of trisomy $\mathrm{X}, 1$ case of monosomy, 2 cases of trisomy XYY, 1 case of trisomy XXY and 1 case of triploidy).

In our study, amongst all abnormal results, prenatal invasive diagnostic tests were able to diagnose fetus with trisomy 21(12), trisomy 13 (2), trisomy 18 (4), Turner's syndrome (4) and 1 each with $6 \mathrm{q}$ addition, $46 \mathrm{XXY}$, triploidy and $6 \mathrm{q}$ addition. There were 2 cases both with translocation $(8,10$ and 6,12$)$ and prominent satellite (15 and 13) and one case with per centric inversion of chromosome nine.

With these tests we also detected one fetus with fetal rubella infection, foetuses with thalassemia major (5), one fetus each affected with Duchene muscle dystrophy, haemophilia and congenital adrenal hyperplasia and two fetus affected with sickle cell disease. There were two cases of fetal anaemia (not due to isoimmunisation) which were managed with neonatal transfusion

There is a constant research going on with considering fetus as a patient. It is because of implying invasive procedure the possibility of treating fetal disease can be carried out. With the help of these invasive procedures, we were able to treat fetal hypothyroidism by instilling in utero levothyroxine through per cutaneous umbilical cord blood sampling. If we look upon the review of literature we find there are few cases reported where there was sonographic diagnosis of a fetal goitre, in a fetus exposed to large doses of propylthiouracil, which was administered to the mother. ${ }^{15}$ The intraamniotic instillation of thyroxine decreased the size of the fetal goitre, and numerous therapeutic amniocenteses permitted continuation of the pregnancy, so a mature infant with a goiter but no airway obstruction was delivered. Amniotic fluid reverse-T3 assays confirmed fetal utilization of the thyroxine.

Indian subcontinent is a harbour of thyroid disease. We very frequently encounter pregnancy with hypothyroidism, hyperthyroidism and thyroid cancer. The intrauterine recognition and treatment of congenital goitres hypothyroidism reduces the obstetric complications associated with large goiters and improves the prognosis for normal growth and mental development of affected foetuses. We have a case report from our centre for a case of fetal goitre diagnosed at 26 weeks of gestation following routine ultrasound examination along with polyhydramnios. ${ }^{16}$ Amniocentesis and fetal blood sampling performed at this time confirmed the presence of fetal hypothyroidism. Since injectable triiodothyronine and thyroxine were not available in India, the fetus was treated with instillation of thyroxine after mixing it with aspirated amniotic fluid. Treatment was performed using a series of intra-amniotic instillation of freshly aspirated amniotic fluid mixed with thyroxine tablet between 26 and 34 weeks. During this period, shrinkage of the fetal goitre, increasing neck flexion and resolution of the polyhydramnios were observed. Following birth, neonatal serum thyroid-stimulating hormone levels were within the normal range but thyroxine was reduced. The baby was started on daily oral thyroxine and on examination at 1year following birth, he appeared clinically and biochemically euthyroid. In the absence of maternal thyroid disease, fetal goitre is extremely rare. There are less than 50 cases previously reported in the english literature to have used intra-amniotic thyroxine injections as a form of treatment. There are no reports of use of thyroxine tablet being used to treat such condition. Hence even in less resourceful settings prenatal procedure can aid in delivering better health care to our patients.

Not only conditions and situation which are apparent through history, clinical presentation and sonography there are also some disease which cannot be definitely commented on unless cytological and biochemical confirmation like diagnosing the origin of fetal ascites or a probable gonadal cyst or for assessment about status of renal function in lower urinary tract obstruction.

Congenital fetal infections remain an area of grey zone unless a definite viral DNA or culture can be obtained from fetal sample. With the help of invasive procedure we confirmed fetal congenital rubella infection and on 
autopsy the fetus did showed congenital cataract and pulmonary stenosis. Hence we could prevent delivery of one fetus with congenital rubella affection.

All these benefits were achieved at a complication rate of $0.029 \%$ and a failure rate of $2.5 \%$, this highlights the need and importance of prenatal diagnostic procedures to be considered as an important part of health care settings.

\section{CONCLUSIONS}

Prenatal diagnosis enables timely medical or surgical treatment of fetal condition before or after birth. It gives a chance to abort a foetus diagnosed to have lethal outcome and prevents psychological trauma of departing at a stage when parents are more attached to their baby. It helps parents to prepare psychologically, socially, financially and medically for a baby with a health problem or disability or for the likelihood of a stillbirth.

The benefits of antenatal procedures and timely detection of fetal abnormalities outweigh the disadvantages like large number needed to undergo and the financial burden. However, at the same time in the present era of noninvasive methods, invasive procedures are definitely not advocated unless indicated.

We realize that prenatal invasive test were effective in diagnosis and preventing birth of abnormal foetus and measures should be taken to lessen the huge financial constraints associated with them so that they can come in reach of common man in most of the developing countries.

\section{ACKNOWLEDGEMENTS}

We acknowledge S.N. Gene lab for their valuable contribution in the study.

\section{Funding: No funding sources}

Conflict of interest: None declared

Ethical approval: Not required

\section{REFERENCES}

1. ACOG Committee opinion number 545:Noninvasive fetal testing for fetal aneuploidy. Obstet Gynecol. 2012;120:1532-4.

2. Evans MI, Wapnar RJ. Prenatal Screening: Incorporating the First Trimester Screening. Seminars in perinatology.2005;29:215-8.

3. Tabor A, Madsen M, Obel E, Philip J, Bang J, Gaard Pedersen. Randomised controlled trial of genetic amniocentesis in 4606 low-risk women. The Lancet. 1986; 327(8493):1287-93.
4. Wapner RJ. Invasive Prenatal Diagnostic Techniques. Seminars in perinatology .2005;29:4014.

5. Brambati B, Terzian E, Tognoni G. Randomized clinical trial of transabdominal versus transcervical chorionic villous sampling methods. Prenat Diagn. 1991;11:285-93.

6. Jackson L, Zachary J, Fowler S, Desnick R, Golbus $\mathrm{M}$, Ledbetter D et al. A randomized comparison of transcervical and transabdomisal chorionic villus sampling. New Engl J Med. 1992;327:594-8.

7. Alfirevic Z, Mujezinovic F, Sundberg K. Amniocentesis and chorionic villus sampling for prenatal diagnosis. Cochrane Database Syst Rev. 2003;(3):CD003252.

8. Nanal R, Kyle P, Soothill PW. A classification of pregnancy losses after invasive prenatal diagnostic procedures: an approach to allow comparison of units with a different case mix. Prenatal Diagnosis. 2003;23:488-92.

9. The Medical Termination of Pregnancy Amendment Act, 2002 (No. 64 of 2002). An Act to amend the Medical Termination of Pregnancy Act, 1971. Government of India, Ministry of Health and Family Welfare.

10. Carnevale A, Hernández M, Reyes R, Paz F, Sosa C. The frequency and economic burden of genetic disease in a pediatric hospital in Mexico City. Am J Med Genet. 1985;20(4):665-75.

11. Kaur A, Singh JR. Chromosomal Abnormalities: Genetic Disease Burden in India. Int J Hum Genet. 2010;10(1-3):1-14.

12. Shawn E, McCandless, Jeanne W, Brunger , Suzanne B. Cassidy. The Burden of Genetic Disease on Inpatient Care in a Children's Hospital. Am J Hum Genet. 2004;74(1):121-7.

13. Nadler HL, Gerbie AB. Role of Amniocentesis in the Intrauterine Detection of Genetic Disorders. N Engl J Med. 1970;282:596-9.

14. Neagos D, Corina R, Camil L. The Importance of Screening and Prenatal Diagnosis in the Identification of the Numerical Chromosomal Abnormalities. Maedica (Buchar). 2011;6(3):179-84.

15. Weiner S, Scharf JI, Bolognese RJ, Librizzi RJ. Antenatal diagnosis and treatment of a fetal goiter. $\mathrm{J}$ Reprod Med. 1980;24(1):39-42.

16. Pradan M, Anand B, Mehrotra M. Management of fetal goitre by intraamniotic instillation of thyroxine tablet : A case report. 2013.

Cite this article as: Kashyap N,Pradhan M, Yadav $\mathrm{S}$, Singh N. Invasive prenatal diagnostic procedures: a developing countries' perspective. Int J Reprod Contracept Obstet Gynecol 2016;5:41-7. 